\title{
Pressemitteilung zur kritischen Stellungnahme von 30 Agrarwissenschaftlern zu den Veröffentlichungen von PD Dr. F. Uekötter zur Rolle der Agrikulturchemie bei der Entwicklung der deutschen Landwirtschaft
}

Online publiziert: 2. September 2011

(C) Springer-Verlag 2011

Der Umwelthistoriker und Privatdozent Dr. Frank Uekötter hat sich in zwei Publikationen sehr ausführlich mit der Entwicklung der landwirtschaftlichen Produktion in Deutschland im Verlauf der letzten 200 Jahre auseinandergesetzt. Die unter 2. genannte Publikation ist zugleich die Habilitationsschrift von Herrn Uekötter.

1. Uekötter, F. (2010): „Im Schatten von Liebig“'In: „Der kritische Agrarbericht 2010“, Hrsg.: AgrarBündnis e. V., 78462 Konstanz Seite 261-265.

2. Uekötter, F. (2010): „Die Wahrheit liegt auf dem Feld“, Verlag Vandenhoeck \& Ruprecht GmbH \& Co.KG, Göttingen, ISBN 978-3-525-31705-1.

Der Autor kritisiert vehement die - nach seiner Meinung - unrühmliche Rolle der Agrikulturchemie als „Hegemonialwissenschaft". Durch die einseitige Konzentration auf die chemische Bodenuntersuchung sei das Basiswissen wichtiger Bereiche, wie Bodenbiologie und Bodenkunde, sträflich vernachlässigt worden, zum Nachteil einer umfassenden Beurteilung der Bodenfruchtbarkeit. Während der Intensivierung der Agrarproduktion nach dem 2. Weltkrieg seien Mineraldünger durchweg exzessiv eingesetzt worden. Über den tatsächlichen Düngebedarf habe man sich nach der Faustregel ,viel hilft viel“" hinweggesetzt. Die extreme Simplifizierung wichtiger Faktoren der Bodenfruchtbarkeit nach dem Prinzip „einfach und unpräzise“ statt „kompliziert und genau“ habe zu einer Art „Blindflug“ der Mineraldüngung geführt. Das alles frei nach der „Methode Liebig“, des Begründers der Agrikulturchemie, „Dreistigkeit siegt“. Nur durch Glück und die enorme Fehlertoleranz der Ackerböden sei es nicht zu einem Kollaps der Bodenfruchtbarkeit in Deutschland gekommen.

Namhafte deutsche Agrarwissenschaftler haben dem Autor inzwischen sachbezogen widersprochen und ihm in Stellungnahmen mangelnde fachliche Kompetenz nachge- wiesen. Dabei wurde vor allem die selektive Auswahl von Literaturzitaten bemängelt, mit denen die teilweise kruden Thesen gestützt werden. Forschungsergebnisse der letzten 3-5 Jahrzehnte, die das Gegenteil belegen, wurden offenkundig gezielt ausgeblendet. Gravierend sind methodische Mängel: Anstatt die geschichtlichen Etappen - und auch die Irrtümer und Fehlentwicklungen - vorurteilslos im Kontext des seinerzeitigen Entwicklungsstandes zu beurteilen - methodische Grundregel eines Historikers (!) -, bewertete Uekötter diese durchweg nur von der „hohen Warte“ unseres heutigen, viel umfangreicheren Wissens über ökologische Kriterien der Pflanzenproduktion. So verfestigt sich der Eindruck, dass es Uekötter vorrangig darum gegangen ist, mit einseitigen, ideologisch geprägten Argumenten zu operieren und zweifellos vorhandene Entwicklungserfolge der Landwirtschaft herabzusetzen. Man muss vermuten, dass Uekötter die begrenzte Sachkenntnis derjenigen Kreise - das ist die große Mehrheit - gezielt ausnutzt, die mit den faktenreichen chemischen und biochemischen Abläufen der Agrarwirtschaft insgesamt nicht vertraut sind.

Eine Gruppe von 30 deutschen Agrarwissenschaftlern hat sich inzwischen mit einer gemeinsamen Stellungnahme an Politik und Medien gewandt, mit dazu beizutragen, dass die Rolle der Agrikulturchemie, ihrer Leistungen und Erfolge für die deutsche Landwirtschaft und die Versorgung der Bevölkerung mit hochwertigen Lebensmitteln in Zukunft sachlich, fachlich belastbar und ohne Polemik und ideologisch geprägte Entstellungen dargestellt und gewürdigt wird.

Karlsruhe, den 19.07.2011

Kontaktadresse: Dr. Rainer Kluge, Bonner Str. 28, 76185

Karlsruhe, oder

Erich Grantzau, Kurze Wende 3, 30926 Seelze 